\title{
The Relationship of Illness Beliefs with Hospital and Emergency Department Utilization in Chronic Obstructive Pulmonary Disease
}

\author{
Himali Weerahandi, MD, MPH' , Juan P. Wisnivesky, MD, DrPH',3, Rachel O'Conor, $\mathrm{MPH}^{4}$, \\ Michael S. Wolf, $P h D^{4}$, and Alex D. Federman, $M D, M P H^{2,3}$
}

'Division of General Internal Medicine and Clinical Innovation, Department of Medicine, NYU School of Medicine, Brooklyn, NY, USA; ${ }^{2}$ Division of General Internal Medicine, Icahn School of Medicine at Mount Sinai, New York, NY, USA; ${ }^{3}$ Division of Pulmonary, Critical Care and Sleep Medicine, Icahn School of Medicine at Mount Sinai, New York, NY, USA; ${ }^{4}$ Division of General Internal Medicine, Feinberg School of Medicine, Northwestern University, Chicago, IL, USA.

KEY WORDS: illness beliefs; medication beliefs; COPD; hospitalization; ED utilization.

J Gen Intern Med 34(6):923-8

DOI: $10.1007 / \mathrm{s} 11606-019-04833-\mathrm{x}$

(c) Society of General Internal Medicine 2019

\section{INTRODUCTION}

Chronic obstructive pulmonary disease (COPD) is the most common cause of potentially preventable inpatient admissions. ${ }^{1,2}$ Avoidable COPD admissions can be prevented with improved self-management. ${ }^{3-10}$ However, while selfmanagement interventions are associated with reduced admissions, studies in this area are heterogeneous in methodology and types of interventions examined, making it challenging to identify key themes to create guidelines to inform effective interventions. $^{3}$

Traditional COPD self-management interventions have primarily focused on improving patients' recognition of symptoms of a COPD exacerbation. These interventions may be made more generalizable if behavioral model framework was applied to them. Behavioral models highlight the role of illness beliefs in patient decision-making about self-management. ${ }^{11}$ Illness beliefs such as cognitive illness representations, emotional representations, and medication beliefs can affect self-management of chronic disease. ${ }^{12-16}$ These beliefs activate behavioral responses and guide coping behaviors, which affect the decisions patients make about how to manage their health. As a result, patients' illness and medicationrelated beliefs can influence disease control. ${ }^{17}$ For example, patients with a mental model of hypertension as a disease controllable through diet and weight management are more likely to have lower systolic blood pressure. ${ }^{18}$ Disease control may in turn affect hospital and emergency department (ED) utilization. Figure 1 illustrates how illness and medication beliefs may affect clinical utilization as a simplified conceptual model; however, the true causal pathways and interactions are

Received March 16, 2018

Revised November 17, 2018

Accepted December 19, 2018

Published online January 31, 2019 unknown. Therefore, we sought to explore the relationship of illness and medication beliefs among patients with COPD with their hospital and ED utilization.

\section{Study Design}

This study was a secondary, cross-sectional data analysis using baseline data from a larger, prospective, multi-site cohort study of COPD patients. The overall objective of the main study was to examine longitudinal associations between cognition, health literacy, and self- management in individuals with COPD. Baseline data for the main study were obtained from interviewer-administered surveys and electronic medical records.

We recruited patients from primary care and pulmonary practices, as well as the inpatient services, of the Mount Sinai Medical Center (East Harlem, New York), Northwestern Memorial Faculty Foundation (Chicago, IL), and Mercy Family Health Center (Chicago, IL) between December 2011 and June 2013. The Institutional Review Boards of the Icahn School of Medicine at Mount Sinai and the Northwestern University Feinberg School of Medicine approved the study.

\section{Study Cohort}

Eligible patients were 55 years and older, English- or Spanishspeaking, and diagnosed with COPD by a health care provider and had a known forced expiratory value $\left(\mathrm{FEV}_{1}\right)$ to forced vital capacity ratio $<0.7$. Excluded patients had a diagnosis of another chronic respiratory disease, or documented history of dementia or other neurological or psychological conditions profoundly affecting cognition (such as advanced Parkinson's disease, stroke, or schizophrenia).

\section{Measures}

The Common Sense Model (CSM) ${ }^{11}$ provided the framework to examine illness and medication beliefs. This model theorizes that patients develop mental representations (beliefs) of 


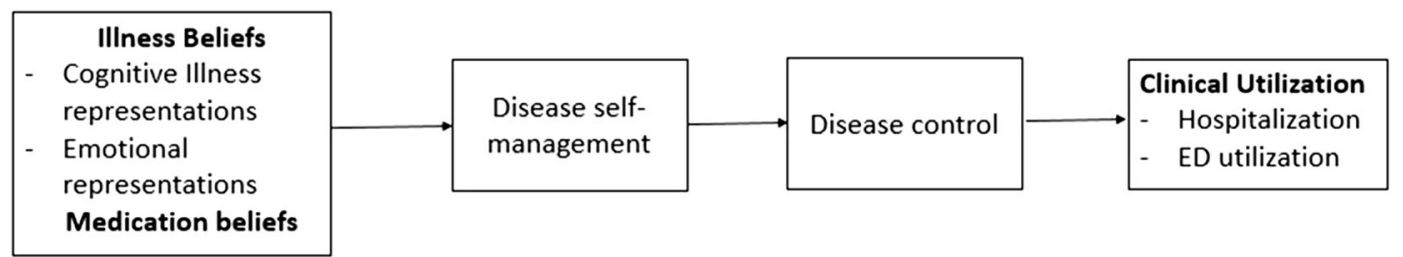

Fig. 1 Conceptual model of how illness and medication beliefs may affect hospital and ED utilization.

their illness based on prior experiences, which can affect health-related behaviors and coping mechanisms. The model posits five domains of cognitive illness representations: identity, timeline, cause, consequences, and control. We focused on timeline and control representations because these beliefs can impact patients' motivation to manage their illness and could be a source of erroneous beliefs where health education can intervene. Timeline ("Will always have COPD") refers to the patient's belief about the chronicity and progressive nature of the illness. Patients with erroneous timeline representations may believe that they only have COPD when experiencing an exacerbation. Control reflects the degree to which patients believe their actions ("COPD is controllable") and treatments ("Treatments can control COPD") can control the illness.

We assessed timeline and control beliefs with the Brief Illness Perceptions Questionnaire (BIPQ), ${ }^{19}$ a validated instrument available in English and Spanish. For timeline, the BIPQ asks the subject to choose the number between 0 and 10 representing how long they think their COPD will continue (very short time to forever). Similarly, for control, the subject is asked to choose the number representing how much control they have over their COPD (where 0 means no control and 10 means complete control) and how much they think their treatment can help their COPD (where 0 means not at all helpful and 10 means extremely helpful). Higher scores indicate a cognitive model consistent with the belief that COPD is a chronic disease.

We assessed medications beliefs with the Beliefs about Medications Questionnaire (BMQ), ${ }^{20}$ a validated instrument with subscales addressing medication necessity beliefs and concerns. The instrument is available in English and Spanish. Responses are rated on a 5-point Likert scale (strongly agree to strongly disagree) and the instrument is scored as the sum of the five responses. Each subscale ranges from 5 to 25. Greater necessity subscale scores indicate beliefs that medications are less necessary. Greater concerns subscale scores indicate higher medication concerns.

We had two outcomes of interest: whether the patient had any hospitalization or ED visits for COPD in the prior 12 months. Due to the large number of medical facilities in these urban environments, this was measured by self-report. These data were obtained by chart review for the $9.2 \%$ and $1 \%$ of patients, respectively, who were missing data for this variable in the baseline survey.

We measured multiple covariates that may impact utilization. Age, gender, race, education, insurance type, marital status, and access to a usual source of care for COPD were self-reported via baseline survey. ${ }^{21}$ Depression was assessed with the Patient Health Questionnaire (PHQ-9). ${ }^{22}$ We defined depression as a PHQ-9 score $\geq 10$. Other co-morbidities, including diabetes, congestive heart failure, atrial fibrillation, and end-stage renal disease, were obtained via self-report and chart abstraction. COPD severity was assessed by spirometry per American Thoracic Society guidelines using a digital spirometer (MidMark Diagnostics Group, Gardena, CA) with $\mathrm{FEV}_{1}$ obtained at the baseline visit.

\section{Statistical Analysis}

Hospitalization and ED utilization were analyzed separately. We compared baseline characteristics of patients with and without history of hospitalization and then ED utilization using the chi-square, $t$ test, or Wilcoxon rank sum test as appropriate. Next, we compared illness beliefs between subjects with and without history of hospitalization and ED visits for COPD, respectively, using the Wilcoxon rank sum test. Finally, we conducted separate multivariable logistic regression models to determine the association of illness beliefs and medication beliefs with hospitalizations and ED visits for COPD, respectively. We created two separate models for each outcome: one that examined illness beliefs and a second that examined medication beliefs. All models controlled for sociodemographics, co-morbidities, predicted $\mathrm{FEV}_{1}$, marital status, level of education, access to care, and insurance. Data were analyzed using SAS version 9.3 (SAS Institute, Cary, NC).

\section{RESULTS}

\section{Study Participation and Patient Characteristics}

We screened 902 potential participants identified by electronic searches; of these, 117 were ineligible and 287 refused. Of the eligible 403 participants, 393 completed the baseline questionnaire. In the overall sample, the mean (SD) age of study participants was 68.1 (8.2) years and $58.5 \%$ were women. The sample was racially and ethnically diverse with $44.0 \%$ identifying as black, non-Hispanic, $36.6 \%$ as white non-Hispanic, and $16.3 \%$ as Hispanic.

Hospitalization data were available for 391 participants and ED utilization data was available for 389 participants. In total, $189(48 \%)$ patients reported having a hospitalization for their COPD (Table 1) and $193(50 \%)$ had a standalone ED visit (Table 2) in the past year. Patients who reported a history of hospitalization for their COPD were more likely to be women, 
Table 1 Subject Characteristics by Hospitalization Use $(n=391)$

\begin{tabular}{|c|c|c|c|}
\hline \multirow[t]{2}{*}{ Characteristic } & Hospitalization, $n=189$ & No hospitalization, $n=202$ & $p$ value \\
\hline & \multicolumn{3}{|l|}{ Median (IQR), mean (SD), or \% $(N)$} \\
\hline Age & $67.4(7.7)$ & $68.8(8.7)$ & 0.199 \\
\hline Female & $65.6 \%(124)$ & $51.5 \%(104)$ & 0.004 \\
\hline \multicolumn{3}{|l|}{ Race } & 0.005 \\
\hline White & $28.0 \%(53)$ & $45.1 \%(91)$ & \\
\hline Black & $49.7 \%(94)$ & $39.1 \%(79)$ & \\
\hline Hispanic & $19.6 \%(37)$ & $12.9 \%(26)$ & \\
\hline Other & $2.7 \%(5)$ & $3.0 \%(6)$ & \\
\hline \multicolumn{3}{|l|}{ Education } & 0.058 \\
\hline Less than 12 years & $26.9 \%(50)$ & $24.3 \%(49)$ & \\
\hline High school graduate & $25.8 \%(48)$ & $18.3 \%(37)$ & \\
\hline Some college & $25.3 \%(47)$ & $23.8 \%(48)$ & \\
\hline College degree or higher & $22.0 \%(41)$ & $33.7 \%(68)$ & \\
\hline Married & $29.1 \%(55)$ & $38.1 \%(77)$ & 0.06 \\
\hline \multicolumn{3}{|l|}{ Insurance } & 0.065 \\
\hline Combined Medicare and Medicaid & $27.7 \%(52)$ & $16.7 \%(33)$ & \\
\hline Medicaid & $19.2 \%(36)$ & $19.7 \%(39)$ & \\
\hline Medicare & $12.2 \%(23)$ & $19.2 \%(38)$ & \\
\hline Private insurance/HMO & $36.2 \%(68)$ & $40.4 \%(80)$ & \\
\hline Self-pay & $4.8 \%(9)$ & $4.0 \%(8)$ & \\
\hline Usual source of care & $80.4 \%(152)$ & $81.2 \%(164)$ & 0.848 \\
\hline \multicolumn{4}{|l|}{ Comorbidities } \\
\hline Atrial fibrillation & $5.8 \%(11)$ & $7.0 \%(14)$ & 0.635 \\
\hline Congestive heart failure & $16.9 \%(32)$ & $6.0 \%(12)$ & 0.001 \\
\hline Diabetes mellitus & $29.1 \%(55)$ & $20.0 \%(40)$ & 0.037 \\
\hline End-stage renal disease & $14.8 \%(28)$ & $9.0 \%(18)$ & 0.076 \\
\hline Predicted $\mathrm{FEV}_{1}^{\mathrm{a}}$ percent & $57.7 \%(22.6 \%)$ & $69.0 \%(24.4 \%)$ & $<0.001$ \\
\hline Depression $\left(\mathrm{PHQ}^{\mathrm{b}} \mathrm{9}^{\mathrm{b}} \geq 10\right)$ & $37.4 \%(70)$ & $20.6 \%(41)$ & $<0.001$ \\
\hline
\end{tabular}

${ }^{a} F E V_{1}$ forced expiratory volume in $1 \mathrm{~s}$

${ }^{b}$ PHQ-9 Patient Health Questionnaire

Table 2 Subject Characteristics by Emergency Department Use $(n=389)$

\begin{tabular}{|c|c|c|c|}
\hline \multirow[t]{2}{*}{ Characteristic } & ED utilization, $n=193$ & $\begin{array}{l}\text { No ED utilization, } \\
n=196\end{array}$ & $p$ value \\
\hline & \multicolumn{3}{|c|}{ Median (IQR), mean (SD), or \% $(N)$} \\
\hline Age & $65.0(12.0)$ & $69.0(11.5)$ & $<0.001$ \\
\hline Female & $65.8 \%(127)$ & $51.5 \%(101)$ & 0.004 \\
\hline \multicolumn{3}{|l|}{ Race } & $<0.001$ \\
\hline White & $23.8 \%(46)$ & $49.5 \%(97)$ & \\
\hline Black & $52.9 \%(102)$ & $35.7 \%(70)$ & \\
\hline Hispanic & $20.2 \%(39)$ & $12.2 \%(24)$ & \\
\hline Other & $3.1 \%(6)$ & $2.6 \%(5)$ & \\
\hline \multicolumn{3}{|l|}{ Education } & $<0.001$ \\
\hline Less than 12 years & $31.6 \%(60)$ & $19.9 \%(39)$ & \\
\hline High school graduate & $27.4 \%(52)$ & $16.3 \%(32)$ & \\
\hline Some college & $22.6 \%(43)$ & $26.5 \%(52)$ & \\
\hline College degree or higher & $18.4 \%(35)$ & $37.2 \%(73)$ & \\
\hline Married & $29.0 \%(56)$ & $38.3 \%(75)$ & 0.054 \\
\hline \multicolumn{3}{|l|}{ Insurance } & 0.003 \\
\hline Combined Medicare and Medicaid & $26.2 \%(50)$ & $18.1 \%(35)$ & \\
\hline Medicaid & $24.1 \%(46)$ & $14.5 \%(28)$ & \\
\hline Medicare & $13.6 \%(26)$ & $18.1 \%(35)$ & \\
\hline Private insurance/HMO & $30.4 \%(58)$ & $46.1 \%(89)$ & \\
\hline Self-pay & $5.8 \%(11)$ & $3.1 \%(6)$ & \\
\hline Usual source of care & $77.7 \%(150)$ & $83.7 \%(164)$ & 0.137 \\
\hline \multicolumn{4}{|l|}{ Comorbidities } \\
\hline Atrial fibrillation & $4.7 \%(9)$ & $7.7 \%(15)$ & 0.22 \\
\hline Congestive heart failure & $17.2 \%(33)$ & $5.6 \%(11)$ & $<0.001$ \\
\hline Diabetes mellitus & $27.1 \%(52)$ & $22.1 \%(43)$ & 0.25 \\
\hline End-stage renal disease & $13.5 \%(26)$ & $10.3 \%(20)$ & 0.318 \\
\hline Predicted $\mathrm{FEV}_{1}^{\mathrm{a}}$ percent & $23.2(59.2)$ & $24.5(67.9)$ & $<0.001$ \\
\hline Depression (PHQ-9 ${ }^{\mathrm{b}} \geq 10$ ) & $38.9 \%(75)$ & $18.4 \%(36)$ & $<0.001$ \\
\hline
\end{tabular}

${ }^{a} \mathrm{FEV} \mathrm{V}_{1}$ forced expiratory volume in $1 \mathrm{~s}$

${ }^{b}$ PHQ-9 Patient Health Questionnaire 
non-white, have depressive symptoms, congestive heart failure, diabetes mellitus, and have a lower mean percent predicted FEV ${ }_{1}$. Similarly, patients with a history of ED use for their COPD were also more likely to be women, non-white, have depressive symptoms, congestive heart failure, and have a lower mean percent predicted $\mathrm{FEV}_{1}$ compared to patients who did not report any history of ED visits for their COPD. Patients with a history of ED use were also more likely to be younger, have combined Medicare \& Medicaid, and have less education.

\section{Relationship Between Health and Medication Beliefs and Utilization}

Timeline scores ("Will always have COPD") were high in all groups (70.7\% said 10 out of 10), indicating most of these patients believed that COPD is a life-long disease. Similarly, treatment control belief score ("Treatments can control COPD") were relatively high in all groups (48.3\% said $\geq 7$ out of 10), indicating most of these patients also believed that treatment helps their COPD. However, patients who had a history of hospitalization had a slightly higher median treatment control belief score than patients who did not have a history of hospitalization $(p=0.001)$ (Table 3 ). Similarly, patients with a history of ED utilization for COPD had a slightly higher median treatment control belief score than patients without a history of ED utilization $(p<0.001)$ (Table 4).

While there was no association between hospitalization and belief about the necessity of medications ("Medications are not necessary") in a bivariate analysis, patients with a history of hospitalization were more likely to have concerns about their COPD medications ("I am concerned about medications") than patients who did not have a history of hospitalization (Table 3). Patients with a history of ED utilization also were more likely to express concerns about these medications but also more likely to believe that their medications were necessary (Table 4).

In multivariable analyses, belief that treatments can control COPD remained associated with COPD hospitalization and ED utilization (OR 1.12, 95\% CI 1.01-1.24, $p=0.04$; OR $1.13,95 \%$ CI $1.02-1.26, p=0.02$, respectively) (Tables 3 and 4). Patients who identified as black $(p=0.03)$, Hispanic $(p=0.02)$, and patients with lower predicted $\mathrm{FEV}_{1}(p<0.001)$ were also more likely to have a history COPD hospital utilization, while younger patients ( $p=0.005$ ), PHQ-9 score $\geq 10$ $(p=0.04), \mathrm{CHF}(p=0.01)$, and lower predicted $\mathrm{FEV}_{1}(p=$ 0.002 ) were more likely to have an ED visit.

In contrast, medication concerns were not significant in separate multivariable models for either hospitalization or ED utilization (Tables 3 and 4). In addition, belief in medication necessity was no longer significantly associated with ED utilization in the multivariable model. In the model examining medication beliefs, race was no longer significant but women $(p=0.01)$ and predicted $\mathrm{FEV}_{1}(p<0.001)$ stayed significantly associated with COPD hospital utilization. In the corresponding multivariable model examining ED utilization, younger patients $(p=0.008)$ and lower predicted $\mathrm{FEV}_{1}(p=0.002)$ remained significantly associated with COPD ED utilization.

\section{DISCUSSION}

In our multi-center study examining illness and medication beliefs in COPD patients, we found that regardless of clinical utilization, these patients do recognize their illness is serious and can be aided by treatment, despite concerns about medication. However, we did find that patients with a history of hospitalization and a history of ED use were slightly more likely to believe in treatment control of their COPD compared with patients without a history of hospitalization or ED use. These findings highlight that common illness misconceptions were not associated with COPD hospitalization or COPD ED utilization.

This contrasts with prior work on illness and medication beliefs in patients with poorly controlled asthma. While the relationship between illness beliefs and hospital and ED utilization for asthma have not been examined specifically, other work has demonstrated that patients with poor medication adherence were more likely to have erroneous asthma related illness beliefs; specifically, they were more likely to believe that they would not always have their asthma. ${ }^{13,15,23}$

While there are similarities in the clinical picture and medical management of asthma and COPD, these patients' illness and medication perceptions may be different. We

Table 3 Unadjusted and Adjusted Associations of Hospitalization with Illness and Medication Beliefs

\begin{tabular}{|c|c|c|c|}
\hline \multirow[t]{2}{*}{$\overline{\text { Beliefs }}$} & Hospitalization, $n=189$ & No hospitalization, $n=202$ & $\overline{\text { Adjusted OR } \text { OR }^{\mathrm{b}}(95 \% \mathrm{CI})}$ \\
\hline & \multicolumn{3}{|l|}{ Median $\left(\mathrm{IQR}^{\mathrm{a}}\right)$} \\
\hline \multicolumn{4}{|l|}{ Cognitive illness representations } \\
\hline I will always have COPD & $10.0(1.0)$ & $10.0(2.0)$ & $1.10(0.99-1.23)$ \\
\hline I have control of my COPD & $6.0(3.0)$ & $6.0(4.0)$ & $0.99(0.90-1.08)$ \\
\hline Treatment can control my COPD & $9.0 * *(3.0)$ & $8.0 * *(5.0)$ & $1.12(1.01-1.24)$ \\
\hline \multicolumn{4}{|l|}{ Medication beliefs } \\
\hline Medications are not necessary & $14.0(8.0)$ & $14.0(7.5)$ & $1.00(0.95-1.06)$ \\
\hline I am concerned about medications & $13.0 *(6.0)$ & $12.0 *(6.0)$ & $1.01(0.94-1.09)$ \\
\hline
\end{tabular}

$* P \leq 05, * * P<.01, * * * P<.001$

${ }^{a} I Q R$ interquartile range

${ }^{b}$ OR odds ratio, CI confidence interval 
Table 4 Unadjusted and Adjusted Associations of Emergency Department Utilization with Illness and Medication Beliefs

\begin{tabular}{|c|c|c|c|}
\hline \multirow[t]{2}{*}{$\overline{\overline{\text { Beliefs }}}$} & ED utilization, $n=193$ & No ED utilization, $n=196$ & $\overline{\text { Adjusted } \mathrm{OR}^{\mathrm{b}}(95 \% \mathrm{CI})}$ \\
\hline & \multicolumn{3}{|l|}{ Median (IQR $\left.{ }^{\mathbf{a}}\right)$} \\
\hline \multicolumn{4}{|l|}{ Cognitive illness representations } \\
\hline I will always have COPD & $10.0(1.5)$ & $10.0(2.0)$ & $1.10(0.99-1.23)$ \\
\hline I have control of my COPD & $6.5(3.0)$ & $6.0(4.0)$ & $0.99(0.90-1.08)$ \\
\hline Treatment can control my COPD & $9.0 * * *(3.0)$ & $8.0 * * *(5.0)$ & $1.13(1.03-1.26)$ \\
\hline \multicolumn{4}{|l|}{ Medication beliefs } \\
\hline Medications are not necessary & $14.0 *(8.0)$ & $16.0 *(7.0)$ & $0.97(0.92 .-1.03)$ \\
\hline I am concerned about medications & $14.0 * * *(6.0)$ & $12.0 * * *(5.0)$ & $1.05(0.97-1.13)$ \\
\hline
\end{tabular}

$* P<05, * * P<.01, * * * P<.001$

${ }^{a} I Q R$ interquartile range

${ }^{b}$ OR odds ratio, CI confidence interval

speculate that this may be because the etiology of COPD is often tied to patient behavior, particularly smoking. ${ }^{24}$ On the other hand, the pathogenesis of asthma is often attributed to genetics or environmental exposures. ${ }^{25}$ In addition, asthma's pathophysiology involves inflammation dominated by eosinophils ${ }^{26}$, making it more responsive to inhaled corticosteroids than COPD, whose inflammation tends to be characterized by a neutrophilic predominance. ${ }^{27}$ The CSM model suggests that a patient's cognitive illness representation framework includes cause, referring to triggers assigned to the health threat onset. These contrasting origins, in addition to disease severity, may create differing cognitive illness representations in these two diseases. As a result, although it may be tempting to apply self-management strategies that have been developed based on what is known about asthma patients' illness beliefs, they may not have the same effectiveness in COPD patients.

Illness and medication beliefs may play a role in mitigating factors that predispose patients towards hospitalization and ED use by influencing self-management behaviors to prevent COPD exacerbation. In particular, we previously reported that patients with concerns about COPD medications were more likely to report non-adherence. ${ }^{28}$ However, in this study, we found that medication concerns are not associated with hospital or ED utilization. It may be that the effect that concern about medications has on self-management behavior alone may not be large enough to influence hospital and ED utilization. Unintentional non-adherence leading to hospital or ED utilization may also confound this relationship if patients with COPD are incorrectly using their inhalers or if their medication regimen is not optimized.

It is important for clinicians to identify how patients' cognitive illness representations may be affected by events such as hospitalization. Identifying beliefs that may be affected by such an event may aid clinicians to target these beliefs that could be susceptible to intervention. For example, while we found that COPD patients overall do believe treatment is helpful to control their COPD, we also found patients with a history of hospitalization or ED utilization were slightly more likely to acknowledge its importance. If a patient has had a recent hospitalization or ED visit, clinicians could potentially capitalize on this event and adherence to medication could be framed around emphasizing patients' belief that medication works for their disease.

This study has several limitations. This was a crosssectional analysis; thus, we cannot comment on whether there is a causal relationship between beliefs and hospitalization or ED visits. There may be a bidirectional effect where beliefs affect experiences, which in return may alter beliefs. Further study should examine these relationships prospectively. In addition, this study was conducted in an urban setting, and may not be generalizable to non-urban settings. It was also limited to patients over the age of 55 and thus may not apply to younger patients. Furthermore, hospitalization and ED use were self-reported and may be inaccurate. Still, these results contribute to the literature describing the cognitive illness model of COPD, which in turn could guide management strategies in COPD.

In summary, this study demonstrated that COPD patients understand they have a chronic disease and that treatment can control their illness, regardless of their history of hospital or ED utilization; we also found erroneous beliefs around disease timeline, control, and medication necessity were not associated with a history of COPD hospitalization or ED use. Consequently, self-management strategies targeting illness beliefs used to impact asthma control may not have the same effectiveness in COPD. Further work is needed to determine whether beliefs can be targeted to improve COPD patient outcomes.

Funding/Support. This work was supported by a grant from the National Heart, Lung, and Blood Institute of the National Institutes of Health under Award Number R01HL105385. Himali Weerahandi is supported by the NYU CTSA grant KL2TR001446 from the National Center for Advancing Translational Sciences, National Institutes of Health.

Corresponding Author: Himali Weerahandi, MD, MPH; Division of General Internal Medicine and Clinical Innovation, Department of Medicine NYU School of Medicine, Brooklyn, NY, USA (e-mail: Himali. weerahandi@nyulangone.org). 


\section{Compliance with Ethical Standards:}

The Institutional Review Boards of the Icahn School of Medicine at Mount Sinai and the Northwestern University Feinberg School of Medicine approved the study.

Conflict of Interest: Juan P. Wisnivesky is a member of the research board of EHE International, has received consulting fees from Merck, UBS, and IMS Health, and was awarded a research grant from GlaxoSmithKline to conduct a COPD study. All remaining authors declare that they do not have a conflict of interest.

Role of the Funder/Sponsor: The NIH had no involvement in the design and conduct of the study; collection, management, analysis, and interpretation of the data; preparation, review, or approval of the manuscript; or decision to submit the manuscript for publication.

Disclaimer: The content is solely the responsibility of the authors and does not necessarily represent the official views of the NIH.

Publisher's Note: Springer Nature remains neutral with regard to jurisdictional claims in published maps and institutional affiliations.

\section{REFERENCES}

1. Fingar KR, Barrett ML, Elixhauser A, Stocks C, Steiner CA. Trends in potentially preventable inpatient hospital admissions and emergency department visits: Statistical brief \#195. In: Healthcare cost and utilization project (HCUP) statistical briefs. Rockville (MD); 2006. NBK338139 [bookaccession].

2. Segal M, Rollins E, Hodges $\mathbf{K}$, Roozeboom M. Medicare-medicaid eligible beneficiaries and potentially avoidable hospitalizations. Medicare Medicaid Res Rev. 2014;4(1):https://doi.org/10.5600/mmrr.004.01. b01. eCollection 2014.

3. Zwerink M, Brusse-Keizer M, van der Valk PD, et al. Self management for patients with chronic obstructive pulmonary disease. Cochrane Database Syst Rev. 2014;3:CD002990. https://doi.org/10.1002/ 14651858.CD002990.pub3 .

4. Blais $\mathbf{L}$, Bourbeau $\mathbf{J}$, Sheehy $\mathbf{O}$, LeLorier $\mathbf{J}$. Inhaled corticosteroids in COPD: Determinants of use and trends in patient persistence with treatment. Can Respir J. 2004;11(1):27-32.

5. Bosley CM, Corden ZM, Rees PJ, Cochrane GM. Psychological factors associated with use of home nebulized therapy for COPD. Eur Respir J. 1996;9(11):2346-2350.

6. Dolce JJ, Crisp C, Manzella B, Richards JM, Hardin JM, Bailey WC. Medication adherence patterns in chronic obstructive pulmonary disease. Chest. 1991;99(4):837-841.

7. Dompeling $\mathbf{E}$, Van Grunsven PM, Van Schayck CP, Folgering $\mathbf{H}$, Molema J, Van Weel C. Treatment with inhaled steroids in asthma and chronic bronchitis: Long-term compliance and inhaler technique. Fam Pract. 1992;9(2):161-166.

8. George J, Kong DC, Thoman R, Stewart K. Factors associated with medication nonadherence in patients with COPD. Chest. 2005; 128(5):3198-3204.

9. James PN, Anderson JB, Prior JG, White JP, Henry JA, Cochrane GM. Patterns of drug taking in patients with chronic airflow obstruction. Postgrad Med J. 1985;61(711):7-10.

10. Rand CS, Nides M, Cowles MK, Wise RA, Connett J. Long-term metered-dose inhaler adherence in a clinical trial. the lung health study research group. Am J Respir Crit Care Med. 1995;152(2):580-588. https://doi.org/10.1164/ajrccm.152.2.7633711.

11. Cameron L, Leventhal H, eds. The self-regulation of health and illness behaviour. New York: Routledge; 2003.

12. Menckeberg TT, Bouvy ML, Bracke M, et al. Beliefs about medicines predict refill adherence to inhaled corticosteroids. J Psychosom Res. 2008;64(1):47-54.

13. Kaptein AA, Klok T, Moss-Morris R, Brand PL. Illness perceptions: Impact on self-management and control in asthma. Curr Opin Allergy Clin Immunol. 2010;10(3):194-199. https://doi.org/10.1097/ACI. Ob013e32833950c1

14. Federman AD, Wisnivesky JP, Wolf MS, Leventhal H, Halm EA. Inadequate health literacy is associated with suboptimal health beliefs in older asthmatics. J Asthma. 2010;47(6):620-626. https://doi.org/10. 3109/02770901003702816.

15. Halm EA, Mora P, Leventhal H. No symptoms, no asthma: The acute episodic disease belief is associated with poor self-management among inner-city adults with persistent asthma. Chest. 2006;129(3):573-580.

16. Kale MS, Federman AD, Krauskopf $\mathbf{K}$, No symptoms, no asthma: The acute episodic disease belief is associated with poor self-management among inner-city adults with persistent asthma. The association of health literacy with illness and medication beliefs among patients with chronic obstructive pulmonary disease. PLoS One. 2015;10(4):e0123937. https://doi.org/10.1371/journal.pone.0123937 .

17. Petrie KJ, Jago LA, Devcich DA. The role of illness perceptions in patients with medical conditions. Curr Opin Psychiatry. 2007;20(2):163167. https://doi.org/10.1097/YCO.0b013e328014a871 .

18. Hekler EB, Lambert J, Leventhal E, Leventhal H, Jahn E, Contrada RJ. Commonsense illness beliefs, adherence behaviors, and hypertension control among African Americans. J Behav Med. 2008;31(5):391-400. https://doi.org/10.1007/s10865-008-9165-4 .

19. Broadbent E, Petrie KJ, Main $\mathbf{J}$, Weinman $\mathbf{J}$. The brief illness perception questionnaire. J Psychosom Res. 2006;60(6):631-637.

20. Horne $\mathbf{R}$, Weinman $\mathbf{J}$, Hankins $\mathbf{M}$. The beliefs about medicines questionnaire: The development and evaluation of a new method for assessing the cognitive representation of medication. Psychology \& Health. 1999;14(- 1): 1. https://doi.org/10.1080/08870449908407311.

21. Centers for Disease Control and Prevention. National health interview survey. 2016.

22. Kroenke K, Spitzer RL, Williams JB. The PHQ-9: Validity of a brief depression severity measure. J Gen Intern Med. 2001;16(9):606-613.

23. Sofianou A, Martynenko M, Wolf MS, et al. Asthma beliefs are associated with medication adherence in older asthmatics. J Gen Intern Med. 2013;28(1):67-73. https://doi.org/10.1007/s11606-012-2160-z .

24. Vogelmeier CF, Criner GJ, Martinez FJ, et al. Global strategy for the diagnosis, management, and prevention of chronic obstructive lung disease 2017 report: GOLD executive summary. Arch Bronconeumol. 2017;53(3):128-149.

25. National Asthma Education and Prevention Program. Expert panel report 3 (EPR-3): Guidelines for the diagnosis and management of AsthmaSummary report 2007. J Allergy Clin Immunol. 2007;120(5, Supplement):S94-S138.

26. Bousquet $\mathbf{J}$, Chanez $\mathbf{P}$, Lacoste $\mathbf{J Y}$, et al. Eosinophilic inflammation in asthma. N Engl J Med. 1990;323(15): 1033-1039.

27. Sutherland ER, Martin RJ. Airway inflammation in chronic obstructive pulmonary disease: Comparisons with asthma. Journal of Allergy and Clinical Immunology. 2003;112(5):819-827.

28. Krauskopf $\mathbf{K}$, Federman AD, Kale MS, et al. Chronic obstructive pulmonary disease illness and medication beliefs are associated with medication adherence. COPD. 2015;12(2):151-164. https://doi.org/10. $3109 / 15412555.2014 .922067$. 